\title{
The influence of microclimate on the development of foot pad dermatitis in broilers
}

\author{
Emanuela Tullo ${ }^{a}$, Giacomo Aletti $^{a}$, Alessandra Micheletti ${ }^{a}$, Giovanni Naldi ${ }^{a}$, Alberto Peña Fernandez ${ }^{b}$, Erik $^{2}$ \\ Vranken $^{b}$, Daniel Berckmans ${ }^{b}$ and Marcella Guarino ${ }^{a}$ \\ ${ }^{a}$ Department of Environmental Science and Policy, Università degli Studi di Milano, Via Celoria, 10, 20133 \\ Milan, Italy \\ ${ }^{b}$ Division Animal and Human Health Engineering, KU Leuven, Kasteelpark Arenberg 30, 3001 Leuven, \\ Belgium
}

\author{
Written for presentation at the \\ $10^{\text {th }}$ International Livestock Environment Symposium (ILES X) \\ Sponsored by ASABE \\ Omaha, Nebraska, USA \\ September 25-27, 2018
}

\begin{abstract}
Footpad dermatitis and lameness are a major welfare concern in broiler chicken farming. In general, footpad lesions are linked to poor environmental conditions. Ulcers that arise from advanced lesions can negatively affect the gait of the birds, with effects on the animal welfare, including, in the worst cases, inability to reach the feed or water.

In this study, the degree of footpad dermatitis and lameness was manually scored on four broiler farms across Europe, as part of an EU-wide welfare assessment programme (Welfare Quality ${ }^{\circledR}$ protocol). Assessment of animal welfare is typically based on manual scoring, which requires a lot of trained manpower/labour.

Precision Livestock Farming (PLF) can combine information technologies into on-line automated tools that can be used to control, monitor and model the behavior of animals and their biological response without stressing, disturbing or handling the animals. The association between the PLF and the Welfare Quality ${ }^{\circledR}$ protocol was investigated to assess whether animal welfare could be better ensured if they were closely monitored with state of the art technology that gives rapid feedback to the farmer. Thus, the aim of this study was to find the association between environmental predisposing factors, measured continuously (such as the indoor temperature and humidity) and leg problems, scored manually during the welfare assessment procedure, in order to find an automated prediction system to detect those lesions. Even if genetics is a heavy predisposing factor for leg disorders, environmental condition could trigger the presence of lesions. Indeed, immobility on litter of poor quality might cause footpad dermatitis affecting the gait of birds. The association between automated control of the environmental conditions and the welfare assessments could be the basis for the development of models and algorithms capable to automatically detect thresholds above which lesions are mostly probable.
\end{abstract}

Keywords. early warning system, health problem, intensive farming, PLF, poultry farming, foot pad dermatitis 


\section{Introduction}

In livestock farming, indoor climate is the main limiting factor to the efficiency of production.

In confined situations, typical of intensive livestock farming, the climate control plays a fundamental role to maintain the health and welfare of animals reared. Indeed, thermal stresses can result in reduced performances, increased morbidity and mortality, with significative losses for farmers (Fournel, Rousseau, \& Laberge, 2017).

To obtain an acceptable level of productivity, the optimal environmental conditions inside the livestock house has to be maintained. In the thermal comfort zone, the energy fraction used by animals to preserve their homeostasis is at a minimum, therefore energy supplied by feed can be used for their growth (meat production) and/or to increase the production of milk or eggs (Mascarenhas et al., 2018). Therefore, when the effective environmental temperature is outside the zone of thermal comfort zone, animal health and the productivity are not at their maximum, with negative consequences for the farm profit.

So, temperature should be carefully controlled and managed since is crucial, and especially for some animal species in the first period of their lives, due to their sensitivity to temperature variations (e.g. chicks and piglets), should be continuously monitored.

Also moisture has to be controlled, because high values of relative humidity may increase the thermal stress of animals causing health problems (Costantino, Fabrizio, Ghiggini, \& Bariani, 2018).

The principles of climate control in barns are focused on temperature and moisture parameters and the ventilation system becomes a key factor to determine optimal conditions for livestock farms. (Curi et al., 2017).

When climate control fails in the goal of maintaining animals in the thermal comfort zone, health and welfare problems can occur, sometimes with serious effects on animals.

This is the case of footpad dermatitis and lameness in poultry farming. Footpad dermatitis (FPD) is characterized as a contact dermatitis on the skin of the foot, both on the central pad and on the toes; the skin turns dark by contact with litter and consequently deep skin lesions can result. Lameness (LMNS) is defined as the inability to use 1 or both limbs in a normal manner. It can vary in severity, ranging from reduced ability or inability to bear weight, to total immobility. They represent a major welfare concern, since lesions may become a gateway for bacterial infections thereby affecting the bird's health. These lesions are provoked by a variety of causes, including the material and thickness of the bedding, the presence of ammonia and moisture in the litter caused by spillage from drinking systems and feces, but in general, foot lesions are linked to poor environmental conditions. (Niels Chr Kyvsgaard, Henrik Bang Jensen, Thorkil Ambrosen, \& Nils Toft, 2013)

Ulcers deriving from advanced lesions can negatively affect the gait of the birds, with potential effects on the welfare of animals, indeed lame birds may also find difficult to reach food and water (Tullo et al., 2017).

In the actual context of intensive farming, Precision Livestock Farming (PLF) could be the guiding concept for the development of an advanced control system based on automatic monitoring, at an appropriate frequency, of environmental, physiological, and behavioral variables (Fournel et al., 2017). PLF can combine information technologies into on-line automated tools that can be used to model, monitor and control the behavior of animals and their biological responses in relation to their environment, without stressing, disturbing or handling the animals.

In this study, the degree of footpad dermatitis and lameness was manually scored on four broiler farms across Europe, as part of an EU-wide welfare assessment program (Welfare Quality ${ }^{\circledR}$ protocol). This kind of assessment is typically based on manual scoring, which requires a lot of trained manpower.

The association between the PLF and the Welfare Quality ${ }^{\circledR}$ protocol was investigated to assess whether animal welfare could be better ensured if they were closely monitored with state of the art technology that gives rapid feedback to the farmer. Thus, the aim of this study was to find the association between environmental predisposing factors, measured continuously (such as the indoor temperature and humidity) and leg problems, scored manually during the welfare assessment procedure, in order to find a climate control system able to limit the incidence of those problems.

\section{Material and methods}

The study was conducted in commercial farms where broilers were reared according to the EU regulation 2007/43/CE. In this study 18 traditional intensive, indoor reared, broiler chicken flocks were inspected between January 2014 to October 2015 in 4 countries, 2 located in the North and 2 located in the South of Europe.

\footnotetext{
The authors are solely responsible for the content of this meeting presentation. The presentation does not necessarily reflect the official position of the American Society of Agricultural and Biological Engineers (ASABE), and its printing and distribution does not constitute an endorsement of views which may be expressed. Meeting presentations are not subject to the formal peer review process by ASABE editorial committees; therefore, they are not to be presented as refereed publications. Publish your paper in our journal after successfully completing the peer review process. See www.asabe.org/JournalSubmission for details. Citation of this work should state that it is from an ASABE meeting paper. EXAMPLE: Author's Last Name, Initials. 2018. Title of presentation. ASABE Paper No. ---. St. Joseph, MI.: ASABE. For information about securing permission to reprint or reproduce a meeting presentation, please contact ASABE at www.asabe.org/permissions (2950 Niles Road, St. Joseph, MI 49085-9659 USA).
}

$10^{\text {th }}$ International Livestock Environment Symposium (ILES X)

Page 3 
Housing and management in the 4 farms involved in the study was very similar. The reared animals were all fast-growing hybrid broilers (Ross 308 or Ross 708) and the microclimate was controlled by the same climate system (Fancom BV, Panningen, The Netherlands). Data regarding FPD, LMNS, and litter quality (LQ) were manually collected on 4 broiler farms. The assessments of animal welfare have been conducted at week 3, 4, and 5 of age of the birds following the Welfare Quality (WQ) protocol on 18 broiler flocks using a five points scoring scale for FPD and a six points scoring scale for LMNS.

Litter quality (LQ) was evaluated to define the housing conditions; this parameter was manually assessed and scored according to the WQ protocol. Assessment scores $\geq 2$ were considered as a threshold for the presence of severe lesions $(0$ and 1), in this way both mild and severe lesions were grouped together, and also the LQ was classified as a binary variable (good or poor) according to this criterion.

During the same rounds, the 24/7 climate control in all the farms considered in the study was completely automated. For this study, the climate variables collected were: temperature inside and outside the barn, relative humidity and maximum and minimum temperature inside. Calibrated relative humidity and temperature sensors were installed on the farm by the PLF technology provider (Fancom BV).

Data from the sensors were collected every 15 minutes on with FarmManager (Fancom BV) and then automatically uploaded and stored into an online data server.

Data of FPD, LMNS and LQ manually assessed with the WQ protocol were merged with climate data continuously collected by farm sensors. All the rounds included in the data collection were used in the statistical analysis.

Broilers are reared under different temperature and humidity ranges according to their age (Aviagen $\left.{ }^{\circledR}, 2014\right)$ and a tightly controlled environment improves animal health, well-being, and production efficiency. Therefore, according to relative humidity (RH) variation, the temperature (T) should be higher at the beginning of the cycle and gradually lowered towards the end of the cycle. To this end, combined thermal comfort indices, such as temperature-humidity index (THI) developed to assess the impact of the thermal environment on thermoregulatory status on several species (Purswell et al., 2012), was used to find a relation between leg disorders and environmental conditions.

The THI was calculated according to the formula (Kibler, 1964):

$\mathrm{THI}=(1.8 * \mathrm{~T}-((1-\mathrm{RH} / 100) *(\mathrm{~T}-14.3))+32)$

In this study the same formula was used to identify the optimal THI ranges for fast growing broilers, based on reference values for T and RH found on the ROSS Management Handbook (Aviagen ${ }^{\circledR}, 2014$ ).

In order to estimate the percentage of time that the broilers spent out of the ranges of thermal comfort (POOC), each hour of the day was classified as " 0 " when the mean hourly THI was included in the ranges expected. On the other hand, it was classified as " 1 " when the mean hourly THI value was out of the optimal thermal situation. In this way, it was possible to sum the hours a day spent out the thermal comfort zone in three reference periods for each round (Day 1-21; Day 1-28; Day 1-35). The results were expressed as a percentage of the total time spent out of the thermal comfort (POOC), before each WQ assessment (performed at week 3, 4,5 of age).

All statistical analyses were conducted with SAS software (SAS Institute). In this study, the binary responses were represented by the presence and absence of FPD and LMNS. Therefore, a mixed-effects logistic regression model using the GLIMMIX procedure with contrasts was applied to investigate the association of POOC, litter quality (LQ), density of birds (DNST, $\mathrm{kg} \cdot \mathrm{m}^{-2}$ ) and age of birds with FPD and LMNS.

The model used was:

$\operatorname{Logit}($ pijklmn $)=\alpha+$ POOC $_{\mathrm{i}}+\mathrm{LQ}_{\mathrm{j}}+\mathrm{DNST}_{\mathrm{k}}+\mathrm{bAGE}_{1}+\mathrm{FR}_{\mathrm{m}}+\mathrm{e}_{\mathrm{ijklm}}$

where pijklmn was the probability of FPD or LMNS occurrence; $\alpha$ was the intercept; POOCi was the fixed effect of the $i$-th class of time spent outside the thermal comfort zone ( $\mathrm{i}=4$ levels: $<40 \%, 40-60 \%, 60-70 \%$ and $>70 \%$ of the time), LQj was the fixed effect of the $\mathrm{j}$-th level of litter quality ( $\mathrm{j}=2$ levels: good and poor), DNSTk was the fixed effect of the kth class of bird density at the moment of each WQ assessment (k= 5 levels: $<20 \mathrm{~kg} / \mathrm{m} 2 ; 20-25 \mathrm{~kg} / \mathrm{m} 2 ; 25-30 \mathrm{~kg} / \mathrm{m} 2 ; 30-35 \mathrm{~kg} / \mathrm{m} 2 ;>35$ $\left.\mathrm{kg} \cdot \mathrm{m}^{-2}\right)$; bAGEl was the regression coefficient for the age $(\mathrm{l}=1$ level, age expressed in days $)$ and $\mathrm{FRm}(\mathrm{m}=18$ levels $)$ was the random effect of the interaction between the farm (4 levels) and the round (14 levels, as the result of the combination of month * year).

The random interaction was used to account for part of the correlations of data within groups in the model. Odds ratios (ORs) and 95\% confidence intervals $(95 \% \mathrm{CI})$ were estimated for each risk factor included in the analysis for FPD and LMNS.

\section{Results and Discussion}

The results of the PROC GLIMMIX to evaluate the effect of risk factors associated with FPD and LMNS are displayed in Table 1.

The results of the present study showed that the percentage of time spent out of the thermal comfort zone (POOC), litter quality (LQ), flock density (DNST, $\mathrm{kg} / \mathrm{m}^{2}$ ) and age of birds were significant predisposing factors for the development of FPD and LMNS in broilers. 
Regarding the FPD, all the effects included in the model resulted highly significant (P-value $<0.01$ ). For LMNS, all the effect considered in the model resulted highly significant (P-value $<0.001$ ), except for the stocking density. The odds ratio associated to the presence (versus the absence) of PFD and LMNS tended to increase proportionally to time spent out of the thermal comfort zone.

The probabilities of having FPD and LMNS changed considerably according to the increase of time spent in an uncomfortable situation. Considering the comparison between POOC classes $(<40 \%, 40-60 \%, 60-70 \%$ and $>70 \%)$, the highest probabilities of FPD and LMNS were obtained when POOC values exceeded the $70 \%$ threshold.

Table 1. Risk factors associated with footpad dermatitis (FPD) and lameness (LMNS).

\begin{tabular}{|c|c|c|c|c|c|c|c|c|c|c|}
\hline & \multicolumn{5}{|c|}{$F P D$} & \multicolumn{5}{|c|}{ LMNS } \\
\hline Parameter & Estimate & Std.Err & P-value $^{a}$ & OR & CI95 & Estimate & Std.Err & $\mathrm{P}_{\text {-value }}{ }^{a}$ & OR & CI95 \\
\hline Intercept & -1.057 & 0.843 & ns & & & 0.370 & 0.730 & ns & & \\
\hline Pooc $^{\text {b }}$ & \multicolumn{5}{|c|}{ reference category $>70 \%$} & \multicolumn{5}{|c|}{ reference category $>70 \%$} \\
\hline$<40 \%$ & -3.317 & 0.477 & $* * *$ & 0.036 & $0.014-0.092$ & -1.778 & 0.278 & $* * *$ & 0.169 & $0.014-0.092$ \\
\hline $40-60 \%$ & -1.993 & 0.358 & $* * *$ & 0.136 & $0.068-0.275$ & -0.718 & 0.211 & $* * *$ & 0.488 & $0.068-0.275$ \\
\hline $60-70 \%$ & -0.946 & 0.330 & $* *$ & 0.404 & $0.212-0.771$ & -0.195 & 0.153 & ns & 0.823 & $0.212-0.771$ \\
\hline Age & 0.054 & 0.015 & $* * *$ & 1.055 & $1.024-1.087$ & 0.011 & $* * *$ & 1.072 & $1.048-1.098$ & $1.024-1.087$ \\
\hline DNST $^{c}$ & \multicolumn{5}{|c|}{ reference category $>35 \mathrm{~kg} / \mathrm{m}^{2}$} & \multicolumn{5}{|c|}{ reference category $>35 \mathrm{~kg} / \mathrm{m}^{2}$} \\
\hline$<20 \mathrm{~kg} / \mathrm{m}^{2}$ & 0.432 & 0.313 & ns & 0.649 & $0.352-1.199$ & -0.323 & 0.282 & ns & 0.724 & $0.352-1.199$ \\
\hline $20-25 \mathrm{~kg} / \mathrm{m}^{2}$ & -1.103 & 0.296 & $* * *$ & 0.332 & $0.186-0.592$ & 0.046 & 0.230 & $\mathrm{~ns}$ & 1.047 & $0.186-0.592$ \\
\hline $25-30 \mathrm{~kg} / \mathrm{m}^{2}$ & -0.154 & 0.234 & ns & 0.857 & $0.542-1.355$ & -0.171 & 0.208 & $\mathrm{~ns}$ & 0.843 & $0.542-1.355$ \\
\hline $30-35 \mathrm{~kg} / \mathrm{m}^{2}$ & -0.640 & 0.313 & $*$ & 0.527 & $0.286-0.973$ & 0.258 & 0.228 & $\mathrm{~ns}$ & 1.294 & $0.286-0.973$ \\
\hline Litter quality & \multicolumn{5}{|c|}{ reference category GOOD } & \multicolumn{5}{|c|}{ reference category GOOD } \\
\hline Poor & -0.390 & 0.121 & ** & 0.678 & $0.535-0.859$ & -0.523 & 0.122 & $* * *$ & 0.593 & $0.467-0.753$ \\
\hline
\end{tabular}

a ***: $\mathrm{P}<0.001 ; * *: \mathrm{P}<0.01 ; *: \mathrm{P}<0.05 ;$ ns: $\mathrm{P}>0.05$

$\mathrm{b}$ POOC: time out of the thermal comfort zone

'DNST: stocking density $\left(\mathrm{kg} / \mathrm{m}^{2}\right)$

The POOC parameter showed how the poor environmental conditions might negatively affect the welfare of the birds. Longer periods spent in uncomfortable situations were associated to increased odds of having FPD and LMNS. Being more than $70 \%$ of time out of the recommended ranges compared to shorter periods $(<40 \%, 40-60 \%, 60-70 \%)$ led to risk probability of having FPD respectively of 28, 7 and 3 times higher. The same comparison was made also for LMNS and showed a similar trend. The risk probabilities of developing LMNS were 6, 2 and 1 times greater, respectively, when the birds spent longer periods $(\geq 70 \%)$ out of the thermal comfort zone compared to shorter periods. This was in line to what was reported by (Martrenchar, Boilletot, Huonnic, \& Pol, 2002), who observed a positive correlation between the prevalence of foot-pad dermatitis and the percentage of moisture in the air. The results were also in accord with (Dawkins, Donnelly, \& Jones, 2004), who found that the number of birds walking well (LMNS $=0$ ) was negatively correlated with the percentage of time that temperature and humidity were outside the breeder's recommended range. Furthermore, improper environmental conditions may result in poor litter quality, commonly associated with increased ammonia burns on the breasts and swollen foot pads (Weaver \& Meijerhof, 1991).

Increased footpad dermatitis was also associated with higher stocking density. Stocking density had a direct effect on FPD and this effect is most likely to be higher at the end of the growing period, when the increased size or weight density of the birds may become more important than their number (Hepworth, Nefedov, Muchnik, \& Morgan, 2010). This was confirmed by the results of this study, in which higher stocking densities were associated to increased probabilities of having FPD. The relation between FPD and stocking density is already known, since greater density of birds would be likely to cause poorer litter quality due to the production of large volume of feces, with a resulting increase in contact dermatitis (Haslam et al., 2007).

On the other hand, stocking density had no significant effect on LMNS, and although it affects chicken welfare, stocking density per se is less important than other factors (Dawkins et al., 2004), since commercial broiler genotypes tend normally to develop LMNS (Nääs et al., 2009).

Our study stressed the importance of litter quality on the development of FPD because litter quality was an important predictor for high FPD and LMNS scores. The general trend of the odds ratio relative to the litter quality indicated that a higher quality (scored 0 and 1 ) was associated to a reduced odd in having lame birds or with lesions. As expected, poor litter quality would led to an increase in contact dermatitis (Kestin, Gordon, Su, \& Sørensen, 2001; N. C. Kyvsgaard, H. B. Jensen, T. Ambrosen, \& N. Toft, 2013).

Modern breeds of broilers have been heavily selected for high juvenile growth rate, breast-meat yield and efficiency of feed conversion, but this has left them vulnerable to welfare problems such as susceptibility to cardio-vascular disease and 
lameness or difficulty in walking (Dawkins \& Layton, 2012; Rizzi, Contiero, \& Cassandro, 2013).

Several studies linked leg disorders to poor environmental conditions (Knowles et al., 2008; N. C. Kyvsgaard et al., 2013; Purswell et al., 2012). Although broilers can tolerate a wide range of relative humidity and still perform efficiently, frequent changes in relative humidity can rapidly and negatively influence litter conditions, that can lead to lowered carcass quality and increased leg and foot abnormalities (Weaver \& Meijerhof, 1991).

\section{Conclusion}

Genetics is a heavy predisposing factor for leg disorders, but environmental conditions could seriously trigger and worsen the presence of lesions. Surely, the high growth rate negatively affects bones development with consequent reduction in birds' activity, but immobility on litter of poor quality might cause footpad dermatitis affecting the gait of birds.

The increase of the age of birds and the time spent outside the thermal comfort zone, associated with a reduced quality of the litter, represent high-risk factors for broilers to incur in severe lesions. Our study stressed the importance of being continuously in optimal thermal conditions.

The association between automated control of the environmental conditions and the welfare assessments could be the basis for the development of models and algorithms capable to automatically detect thresholds above which lesions are mostly probable. PLF systems have been widely used to monitor the behaviour, the position and the activity, the growth and the welfare of broiler chickens, but can be effectively used to control the indoor climate. The advantage of these monitoring systems is that more information can be collected continuously without stressing, disturbing or handling the animals. Moreover, the combination of inexpensive technology and relative simple statistical analysis of environmental parameters can give valuable information quickly and easily, having potential for wide application in animal husbandry.

Thus, it is the time to develop a new generation of environmental control system, continuously recording data collected by electronic sensors and instruments, to be used to assess the effectiveness of certain controls on the modification of ambient conditions on the welfare of the broilers, reducing the costs and the response time in case of problems in the farm.

\section{References}

Aviagen ${ }^{\circledR}$. (2014). ROSS Broiler management handbook. Retrieved from

Aydin, A., Cangar, O., Ozcan, S. E., Bahr, C., \& Berckmans, D. (2010). Application of a fully automatic analysis tool to assess the activity of broiler chickens with different gait scores. Computers and Electronics in Agriculture, 73(2), 194-199.

Costantino, A., Fabrizio, E., Ghiggini, A., \& Bariani, M. (2018). Climate control in broiler houses: A thermal model for the calculation of the energy use and indoor environmental conditions. Energy and Buildings, 169, 110-126. doi:https://doi.org/10.1016/j.enbuild.2018.03.056

Curi, T. M. R. d. C., Conti, D., Vercellino, R. d. A., Massari, J. M., Moura, D. J. d., Souza, Z. M. d., \& Montanari, R. (2017). Positioning of sensors for control of ventilation systems in broiler houses: a case study. Scientia Agricola, 74(2), 101-109.

Dawkins, M. S., Cain, R., \& Roberts, S. J. (2012). Optical flow, flock behaviour and chicken welfare. Animal Behaviour, 84(1), 219-223. doi:10.1016/j.anbehav.2012.04.036

Dawkins, M. S., Donnelly, C. A., \& Jones, T. A. (2004). Chicken welfare is influenced more by housing conditions than by stocking density. Nature, 427(6972), 342-344. doi:10.1038/nature02226

Dawkins, M. S., \& Layton, R. (2012). Breeding for better welfare: genetic goals for broiler chickens and their parents. Animal Welfare, 21(2), 147-155. doi:10.7120/09627286.21.2.147

Fontana, I., Tullo, E., Butterworth, A., \& Guarino, M. (2015). An innovative approach to predict the growth in intensive poultry farming. Computers and Electronics in Agriculture, 119, 178-183. doi:10.1016/j.compag.2015.10.001

Fontana, I., Tullo, E., Scrase, A., \& Butterworth, A. (2016). Vocalisation sound pattern identification in young broiler chickens. Animal, 10(9), 1567-1574. doi:10.1017/S1751731115001408

Fournel, S., Rousseau, A. N., \& Laberge, B. (2017). Rethinking environment control strategy of confined animal housing systems through precision livestock farming. Biosystems Engineering, 155, 96-123.

Haslam, S. M., Knowles, T. G., Brown, S. N., Wilkins, L. J., Kestin, S. C., Warriss, P. D., \& Nicol, C. J. (2007). Factors affecting the prevalence of foot pad dermatitis, hock burn and breast burn in broiler chicken. Br Poult Sci, 48(3), 264-275. doi:10.1080/00071660701371341

Hepworth, P. J., Nefedov, A. V., Muchnik, I. B., \& Morgan, K. L. (2010). Early warning indicators for hock burn in broiler flocks. Avian Pathology, 39(5), 405-409. doi:10.1080/03079457.2010.510500

Kestin, S. C., Gordon, S., Su, G., \& Sørensen, P. (2001). Relationships in broiler chickens between lameness, liveweight, growth rate and age. Veterinary Record, 148(7), 195-197. doi:10.1136/vr.148.7.195

Kibler, H. (1964). Thermal effects of various temperature-humidity combinations on Holstein cattle as measured by eight physiological responses. MO Agr. Exp. Res. Bull, 862.

Knowles, T. G., Kestin, S. C., Haslam, S. M., Brown, S. N., Green, L. E., Butterworth, A., . . Nicol, C. J. (2008). Leg disorders in broiler chickens: prevalence, risk factors and prevention. PloS one, 3(2), e1545. 
doi:10.1371/journal.pone.0001545

Kyvsgaard, N. C., Jensen, H. B., Ambrosen, T., \& Toft, N. (2013). Temporal changes and risk factors for foot-pad dermatitis in Danish broilers. Poultry Science, 92(1), 26-32.

Kyvsgaard, N. C., Jensen, H. B., Ambrosen, T., \& Toft, N. (2013). Temporal changes and risk factors for foot-pad dermatitis in Danish broilers. Poult Sci, 92(1), 26-32. doi:10.3382/ps.2012-02433

Littell, R. C., Stroup, W. W., Milliken, G. A., Wolfinger, R. D., \& Schabenberger, O. (2006). SAS for mixed models: SAS institute.

Martrenchar, A., Boilletot, E., Huonnic, D., \& Pol, F. (2002). Risk factors for foot-pad dermatitis in chicken and turkey broilers in France. Prev Vet Med, 52(3-4), 213-226. doi:http://dx.doi.org/10.1016/S0167-5877(01)00259-8

Mascarenhas, N. M. H., da Costa, A. N. L., Pereira, M. L. L., de Caldas, A. C. A., Batista, L. F., \& Andrade, E. L. G. (2018). Thermal conditioning in the broiler production: challenges and possibilities. Journal of Animal Behaviour and Biometeorology, 6(2), 52-55.

Nääs, I., Paz, I. C. d. L. A., Baracho, M., Menezes, A., Bueno, L., Almeida, I., \& Moura, D. (2009). Impact of lameness on broiler well-being. The Journal of Applied Poultry Research, 18(3), 432-439.

Peña Fernández, A., Tullo, E., Exadaktylos, V., Vranken, E., Guarino, M., \& Berckmans, D. (2015). Broiler activity and distribution as behavior-based welfare indicators.

Purswell, J. L., Dozier III, W. A., Olanrewaju, H. A., Davis, J. D., Xin, H., \& Gates, R. S. (2012). Effect of temperaturehumidity index on live performance in broiler chickens grown from 49 to 63 days of age. Paper presented at the 2012 IX International Livestock Environment Symposium (ILES IX).

Rizzi, C., Contiero, B., \& Cassandro, M. (2013). Growth patterns of Italian local chicken populations. Poult Sci, 92(8), 2226-2235. doi: $10.3382 /$ ps.2012-02825

Tullo, E., Fontana, I., Peña Fernández, A., Vranken, E., Norton, T., Berckmans, D., \& Guarino, M. (2017). Association between environmental predisposing risk factors and leg disorders in broiler chickens. Journal of Animal Science, 95(4), 1512-1520.

Weaver, W. D., Jr., \& Meijerhof, R. (1991). The effect of different levels of relative humidity and air movement on litter conditions, ammonia levels, growth, and carcass quality for broiler chickens. Poult Sci, 70(4), 746-755. 